\section{Effects of eye movements on the rate of discovery and the vividness of visual images:}

\author{
S. M. HALE and H. M. SIMPSON† \\ Carleton University, Ottawa, Ontario, Canada
}

Two studies investigated the effects of eye movements on the rate of discovery and the vividness of visual images. Eye movements were manipulated by having three conditions: (1) The Ss were instructed to make eye movements while generating images to noun pairs; (2) the Ss were instructed not to make eye movements, but to think about making eye movements while generating images to noun pairs;(3) the Ss were instructed not to make eye movements and not to think about making eye movements while generating images to noun pairs. In addition, the ease of generating images was manipulated by using noun pairs that differed in their image-evoking capacity; five of the noun pairs consisted of high imagery-evoking nouns and five consisted of low imagery-evoking nouns. The two experiments were similar, with the exception that a between-groups design was used in Experiment 1, whereas Experiment 2 employed a within-Ss design. The results of both experiments showed highly significant effects of noun-pair type on both the rate of discovery and the vividness of images, with the fastest and most vivid images occuring to the high-imagery noun pairs. The effects of the eye-movement conditions on the rate of discovery and the vividness of the images were not significant in either experiment, and these findings are discussed in terms of the relationship of ocular activity to imagery.

Studies have shown that eye movements accompany visual imagery (e.g., Lorens \& Darrow, 1962), and several authors have interpreted this finding as evidence that ocular activity is directly related to the visual content of the imagery (e.g., Deckert, 1964; Zigmund, 1964; Hebb, 1968). Lorens and Darrow (1962) recorded eye movements, skin conductance level, EEG, and heart rate during a 15 -min relax period followed by mental multiplication and found a significant increase in e y e-moveme $n t$ rate (e ye movements/minute) during the multiplication period, but found no correlation between eye-movement rate and the other psychophysiological indices. These data suggest that rapid ocular movement is a concomitant of mental multiplication but is independent of levels of arousal as reflected by the other physiological measures. Lorens and Darrow postulated that the increase in eye-movement rate reflected scanning of the visual images formed by $S s$ during calculation. Further evidence directly relating eye movements to the content of visual imagery is given by a study (Deckert, 1964) in which Ss, while attempting to imagine a beating pendulum, developed

\footnotetext{
*This research was based on a thesis by the first author submitted to the Graduate School of Carleton University in partial fulfillment of the requirements for the MA degree. The study was supported by a National Research Council of Canada Bursary to the first author and by Grant APA-285 from the National Research Council of Canada to the second author.

t Requests for reprints should be sent to H. M. Simpson, Department of Psychology, Carleton University, Ottawa 1, Ontario, Canada.
} Nevertheless, Hebb has recently argued $(1968$, p. 470$)$ that eye movements are not only a concomitant of imagery but a necessary condition for images to occur. Hebb provides mainly anecdotal evidence to support his statement, which is based on his theoretical analysis of imagery within the conceptual framework of the theory of cell assemblies (Hebb, 1949). In his original formulation of this theory, Hebb stated that eye movements were not essential to perception or to imagery. He emphasized that a stable, clear, and effective perception or image was more possible with eye movement than without, and that "imaginary" eye movements or subliminal activations of the motor system also facilitated perception and imagery but were not essential or sufficient to it. $\mathrm{He}$ now apparently believes (Hebb, 1968) that eye movements are indeed essential to visual imagery.

The principal experimental evidence for the controversy described above has come from studies in which eye movements have been the dependent variable. Eye movements have, therefore, been identified only as a concomitant of a variety of tasks, including those which presumably involve visual imagery. A logical step, then, is to manipulate eye movements as an independent variable and determine the effects, if any, of these manipulations on visual imagery. 1 Such was the purpose of the present two experiments. The experiments were similar, with the exception that a between-groups design was used in Experiment 1, whereas Experiment 2 employed a within-Ss design.

The experimental paradigm basically involved a comparison of the effects of eye movements on imagery, i.e., a condition in which Ss made eye movements while generating images to noun pairs, as well as a condition in which Ss did not make eye movements. Since it has been suggested recently (Hebb, 1968) that imagery can occur in the absence of overt eye movements, provided $S$ imagines the appropriate ocular activity, a third condition was included in the experiment. In this condition $S$ was instructed not to make overt eye movements, but to imagine making them while generating images to noun pairs. The ease or difficulty of obtaining an image was manipulated by having $\mathrm{S}$ generate imaginal mediators to noun pairs that had been shown previously (e.g., Yuille \& Paivio, 1968) to elicit images differentially. If indeed eye movements are an integral part of imagery, then both the rate at which $S$ can generate an image, as well as the clarity of the image, should be affected by eye movements. Accordingly, the latency and the vividness of the images generated by $S$ were determined in each case.

\section{EXPERIMENT 1}

Method

Subjects. The Ss were 45 female volunteers from Carleton University whose mean age was 18.4 years. The Ss were able to fixate a $1-\mathrm{cm}$-square target at a distance of $1.22 \mathrm{~m}$ without the aid of glasses. The 45 Ss were assigned randomly to one of the three treatment conditions.

Design. The three treatment conditions were as follows: (1) The Ss were instructed to make eye movements during the imagery task (EM group); (2) the Ss were told not to make eye movements, i.e., to fixate on a target but to think about or imagine making eye movements during the imagery task (TEM group); (3) the Ss were told not to make eye movements and not to think about making eye movements during the imagery task (NEM group). In addition to the three independent groups, there were two levels of a within-S variable, 
namely, the image-evoking capacity of noun pairs which were either high imagery-evoking pairs (Hi I) or low imagery-evoking pairs (Low I).

Stimulus material. A pool of $\mathrm{HiI}$ and Low I nouns, matched on meaningfulness and frequency, were selected by Es from a list of 925 nouns previously rated on these dimensions (Paivio, Yuille, \& Madigan, 1968). A Hi I noun was defined as one having a mean I rating greater than 6.00 (on a 7-point imagery scale), while a Low I noun was defined as one having a mean I rating of less than 3.00 . From this pool, 10 Hi I nouns and 10 Low I nouns were selected randomly and paired to give $5 \mathrm{Hi} \mathrm{I}$ noun pairs and 5 Low I noun pairs. The resulting 10 noun pairs were arranged in three different random orders, with the stipulation that no more than $2 \mathrm{Hi} \mathrm{I}$ or 2 Low I pairs occur in sequence. In addition, for purposes of preexperimental training, 20 nouns were selected randomly by the Es from the list of 925 nouns (with the stipulation that they had not been selected for the experimental list of $\mathrm{Hi} \mathrm{I}$ and Low I noun pairs).

In each of the three experimental word lists, the noun pairs were separated by the word "relax," so that the order of stimuli was: noun pair, "relax," noun pair, and so on. The interval between the noun pair and "relax" was $20 \mathrm{sec}$, while the interval between "relax" and the noun pair was $10 \mathrm{sec}$. The three lists of stimuli were tape-recorded for presentation to the Ss.

Apparatus. Eye movements were monitored by means of Lexington cup electrodes attached to the outer canthus of each eye. The electrooculographs (EOGs) were amplified and recorded on a Grass Model 7 polygraph. In order to minimize movement artifacts in the EOGs, $S$ was seated in a dental chair with her head resting in a head clamp. A relatively uniform field of vision was provided by placing a projection screen, which was tilted in the same angle as S's head, $1.22 \mathrm{~m}$ in front of $S$. The screen was illuminated by a slide projector that was located above and behind S's head. This enabled the rest of the room to be darkened so as not to distract $S$ from the task. A removable fixation target, as well as a removable calibration chart, were fastened to the center of the screen.

A tape recorder was coupled to the driver amplifier of one channel of the polygraph, so that stimuli presented to $S$ were indicated on the chart paper. Another channel of the polygraph was occupied by a time marker, which was also interfaced with a button located by $S$ 's right hand.

Procedure. The $\mathrm{Ss}$ were tested individually. Each $S$ was seated in the dental chair, and the electrodes were

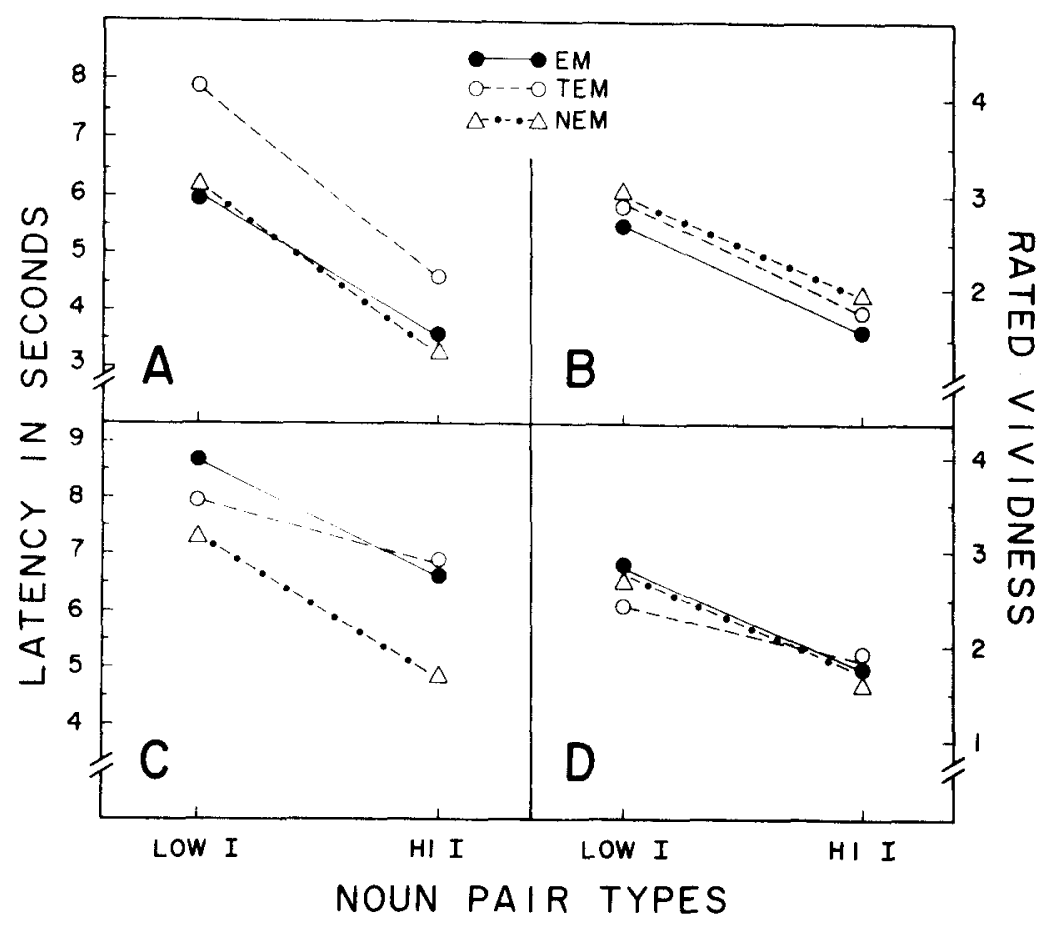

Fig. 1. Mean latencies and rated vividness of mediating images generated to high- and low-imagery noun pairs for Ss in three eye-movement conditions. Experiment 1 (A and B) involved a between-groups design, whereas Experiment 2 (C and D) was within-Ss.

applied to the outer canthus of each eye. The sensitivity of the polygraph was adjusted for each $S$ by having $S$ fixate certain points on the calibration chart in order to obtain a setting that would give a maximum pen deflection on the polygraph for a minimum amount of pen drift or, in other words, a maximum signal/noise ratio for that $S$. The $S$ was then given the general instructions common to all Ss. Basically, S was told that a series of word pairs would be presented one pair at a time on the tape recorder and that her task was to form an image that linked the pair, that is, to imagine some object, event, or situation that incorporated the images suggested by the two words. The $S$ was told to press the button when she had the mediating image and to verbally rate on a 5-point scale the vividness or clarity of that image ( 1 on the scale indicated a perfectly clear, vivid image). In addition, $\mathrm{S}$ was given specific instructions to move or not move her eyes while generating an image, depending on the treatment condition to which she had been assigned. The $\mathrm{S}$ was then given 10 practice trials to ensure that she understood and was able to comply with the task requirements. Prior to each trial, $S$ was reminded of her eye-movement condition. Following the practice items, the experimental session began.

During the experimental trials, the vividness ratings reported by $S$ were recorded by $\mathrm{E}$ on the polygraph chart paper. In this way all the data recorded, that is, EOGs, imaginal latencies, and vividness ratings were contained on the chart paper for each $S$.

\section{Results}

Imaginal latencies were determined for each word pair for each $S$ by measuring on the polygraph chart paper the amount of time that elapsed from the offset of the last noun in the pair to the onset of the buttonpress. These scores were then averaged across noun-pair types, separately for $\mathrm{Hi} \mathrm{I}$ and Low I pairs, yielding two mean imaginal latencies for each S. A 3 by 2 analysis of variance (ANOVA) compared the three groups (EM, TEM, and NEM) and the two types of noun pairs $(\mathrm{Hi} \mathrm{I}$ and Low I) on the mean ${ }^{2}$ imaginal latencies. The means for Low I and Hi I noun pairs are presented graphically for the three groups in Fig. 1A. ${ }^{3}$ The ANOVA showed no significant main effect of groups (e ye-movement conditions), $F(2,42)=1.77$, and no significant interaction of groups with noun-pair types, $F(2,42)=.31$. However, there was a highly significant effect of noun-pair types, $F(1,42)=39.60, p<.01$, and, as shown in Fig. 1A, the imaginal latencies to Hi I noun pairs were shorter (faster) than the imaginal latencies to Low I noun pairs.

The vividness data were treated in a similar manner to the latency data and then analyzed using a 3 by 2 ANOVA. The appropriate means 4 are shown graphically in Fig. 1B. The ANOVA indicated a highly 
significant effect of noun-pair types, $F(1,42)=99.84, p<.01$. As shown in Fig. 1B, the vividness ratings to the $\mathrm{Hi} \mathrm{I}$ pairs were lower (clearer) than the vividness ratings to Low I pairs. Neither the main effect of groups $[F(2,42)=1.13]$ nor the interaction of groups with noun pair types $[F(2,42)=0.08]$ was significant.

The EOGs for the EM group were examined to determine the frequency of eye movements during the imaging period for each word pair for each $S$. The total number of eye movements during an imagining period was calculated and that number was divided by the imaginal latency to obtain the frequency of eye movements (eye movements per second) during the period in which $S$ was generating an image. The eye-movement frequencies were averaged across noun pairs for each $S$. Separate Pearson product-moment correlations were done to examine the relationship between the frequency of eye movements and the median imaginal latency scores, as well as between frequency and median vividness ratings. None of the correlations proved to be significant, and they were not all in the same direction.

The Ss did not always comply with the experimental instructions in that sometimes eye movement occurred during an imaging period, when the $S$ had been instructed to fixate the target. The EOGs for the TEM and NEM groups were examined to determine to what extent eye movements had in fact occurred during the imaging periods. For the TEM groups, eye movements were found to occur in 13 out of $75(17 \%)$ of the Low I imaging periods and in 7 out of $75(13 \%)$ of the $\mathrm{HiI}$ imaging periods. For the NEM group these values were $12 \%$ and $5 \%$ for the Low I and $\mathrm{Hi}$ I imaging periods, respectively. The data for Ss in the TEM and NEM groups who had failed to comply with the task instructions were reexamined. The latency scores and vividness ratings from the imaging periods during which eye movements had occurred in error were eliminated, and the median imaginal latencies and median vividness ratings were recalculated on the basis of the remaining scores. The group averages were then calculated using the revised median latency scores and vividness ratings for those Ss who had failed to comply with the instructions. These revised group averages differed very little from the original group means. Thus, the latency and vividness scores do not appear to have been affected by those instances when eye movements occurred in error.

The results for this experiment will be discussed in conjunction with the results for Experiment 2.

\section{EXPERIMENT 2}

In the first experiment there was a high degree of response variability among Ss. This was especially true with respect to imaginal latencies, in that some Ss consistently responded in 1 or $2 \mathrm{sec}$ to both Low I and Hi I noun pairs, while other Ss consistently had imaginal latencies of 10 or $12 \mathrm{sec}$. In other words, some Ss seemed to be "fast imagers" and other Ss to be "slow imagers," and this may have masked the treatment effects of eye-movement manipulation. It is still possible, therefore, that eye movements facilitate image generating and that this effect did not show in Experiment 1 due to the variability of responses among Ss. If this were so, manipulating the three eye-movement conditions in a within-Ss design should result in significant differences in both the imaginal latencies and the vividness ratings between the eye-movement conditions. If eye movements are facilitative to image generating, then the imaginal latencies and the vividness ratings should be ranked for the three conditions in the order EM, TEM, NEM, with the shortest latencies and the most vivid images occurring in the EM condition.

\section{Method}

Subjects. The Ss were 18 female volunteers from the introductory psychology class at Carleton University whose mean age was 19.2 years.

Design. The design was basically the same as in Experiment 1, except that each $S$ received all three eye-movement conditions. The order of eye-movement conditions was determined randomly for each S (see Gaito, 1958, 1961).

Stimulus material. The Hi I and Low I nouns were selected by $E_{s}$ in a manner similar to that described in Experiment 1. The only difference was that a Low I noun was defined as one having a mean I rating of less than 4.20 . It was necessary to raise the upper limit for defining Low I nouns from that used in Experiment 1 in order to obtain the greater number of noun pairs required. Three separate lists were required for this experiment, a different one for each of the three eye-movement conditions.

Apparatus. The apparatus was the same as that used in Experiment 1.

Procedure. The procedure was basically the same as that in Experiment 1, except that in the present experiment each $S$ received all three eye-movement conditions. Each condition was preceded by specific instructions and practice trials to ensure that the $S$ understood the task requirements.

\section{Results}

Latency data. Imaginal latencies were determined for each noun pair for each $S$. The median latencies were then calculated separately for Hi I and Low I noun pairs for each $S$ for each eye-movement condition. These data were analyzed using a 3 by 2 ANOVA with repeated measures on both variables. The group means are presented graphically in Fig. 1C. The only significant effect that emerged from the analysis was that of noun pair types, $F(1,34)=16.08, p<.01$, and, as shown in the figure, the imaginal latencies to $\mathrm{Hi}$ I noun pairs were faster than the imaginal latencies to Low I noun pairs.

Vividness data. The vividness ratings were summarized in the same manner as the latency data and then analyzed, using a 3 by 2 ANOVA with repeated measures on both variables. The appropriate means are presented graphically in Fig. 1D. As with the latency data in this experiment, and consistent with the results from Experiment 1, the only significant effect was that of noun pair types, $F(1,34)=31.67, p<.01$, with the most vivid images being evoked by the $\mathrm{Hi}$ I noun pairs.

EOG data. The EOGs were examined as in Experiment 1 to determine the extent to which Ss did or did not comply with the task requirements. The data for each $S$ who had failed to comply with the task requirements were revised, first by eliminating the scores of imaging periods in which eye movements had occurred, and then by calculating the median latencies and median vividness ratings on the basis of the remaining scores. As in Experiment 1, the revised data differed little from the original data in that the magnitude of the change in score was not large, and there was no consistent direction of change.

\section{DISCUSSION}

The results of the present two experiments do not support the hypothesis that eye movements are necessary for visual imagery or even that eye movements enhance visual imagery. In both experiments $S s$ indicated by the buttonpress that they were able to generate images under a condition where overt eye movements did not occur (the NEM condition). Furthermore, the analyses of variance showed no significant differences in latency scores or vividness ratings for the three groups (EM, TEM, and NEM) in either Experiment 1 or 2. The failure to find an eye-movement effect suggests that ocular activity is neither essential nor necessarily facilitative to visual imagery. One possible objection to this finding is that eye movements of less than $1 \mathrm{deg}$ could have occurred in the TEM and NEM 
onditions (which would not have been etected on the EOG) and that these eye lovements would be sufficient to facilitate nagery, thereby eliminating any ifferences among the three conditions. lowever, this possibility appears unlikely or several reasons. First, the eye lovements that did occur during the naging periods of the EM groups were Ifficiently large to be recorded on the .OG and, in fact, were most often lovements of more than $5 \mathrm{deg}$. Second, Ss lere given a $1-\mathrm{cm}$-square target to fixate nd indicated in postexperimental uestioning that they experienced no ifficulty in fixating this target. However, he EOGs for Ss in the TEM and NEM roups occasionally showed eye lovements that were made in error, but he Ss were aware of this when it appened. Furthermore, when an eye lovement was recorded during an imaging reriod in the TEM and NEM groups and he latency and vividness scores for that maging period were removed from that S's cores, there was little effect on the mean core for that $S$ or for that group. If eye novements do in fact facilitate imagery, me would expect shorter latencies and nore vivid ratings for the imaging periods $n$ which the eye movements occurred, and emoval of the scores from those imaging seriods should result in a higher mean atency (longer) and a higher (less vivid) rividness rating. The fact that this did not Iappen suggests that the presence of eye novements does not affect the latency or rividness of images, and it further ubstantiates the findings from the analyses of variance between the groups.

In addition, the correlational analyses lid not show any significant relationship setween the frequency of eye movements ind the vividness of imagery.

The data from the two experiments were inalyzed in several different ways (see Vote 2) to ensure that the lack of significant results (with respect to the zye-movement effect) was not due to the jarticular statistical tests involved. This, Jlus the fact that the noun pair variable was highly significant in all analyses, enders unlikely the possibility that the esults of the experiment were due to artifacts of statistical analyses and itrengthens the suggestion that eye novements are not essential or necessarily acilitative to generating visual images to zoun pairs.

The results of both experiments, lowever, clearly show that the image-evoking capacity of noun pairs is a potent variable affecting the imaginal latency and vividness of images. There were highly significant differences in both experiments between the latency scores to
Low I and to Hi I noun pairs, and between the vividness scores to Low $\mathrm{I}$ and $\mathrm{Hi} I$ noun pairs. This supports the findings of other researchers (e.g., Yuille, 1965; Paivio, 1966; Simpson \& Paivio, 1968), that imaginal latencies are longer to Low I nouns than to Hi I nouns and that the images evoked by Low 1 nouns are less vivid than those evoked by $\mathrm{Hi}$ I nouns.

What then do the findings mean in terms of the nature of the relationship of eye movements to visual imagery? The results especially cast doubt on Hebb's argument (1968, p. 470) that eye movements are essential to visual imagery. Ss were able to form images in the NEM condition. In addition, it was no harder for Ss to form clear images with fixation of gaze while imagining the eye movements than it was to form clear images when eye movements were made freely. It may be argued that these results are an artifact due to Ss not complying with the task demands. That is, it is impossible to be sure that $S s$ in the TEM group did imagine the appropriate eye movements or that $S s$ in the NEM group did not imagine eye movements. However, the Ss reported that they were able to comply with the instructions without any difficulty. If one argues that subjective reports are not valid in this respect, then Hebb's hypothesis becomes difficult to test. The experimental evidence then does not support Hebb's hypothesis, nor, of course, does it reject it entirely, since the data are limited to the generating of images to word pairs. The findings do, nevertheless, cast doubt on the validity of his statement and indicate the necessity for further research.

More generally, the results do not show that eye movements are unrelated to visual imagery but do show that, with some stimuli, eye movements are not a necessary and sufficient condition to generate images. Ocular activity may in some cases be intimately related to imagery, as indeed seems to be the case with images of a moving stimulus.

\section{REFERENCES}

AMADEO, M., \& SHAGASS, C. Eye movements, attention and hypnosis. Journal of Nervous \& Mental Disease, 1963, 136, 139-145.

ASHER, E. J., \& ORT, R. A. Eye movements as a complex indicator. Journal of General Psychology, 1951, 45, 209-217.

BALDRIDGE, B. J., WHITMAN, R. M., DRAMER, M., \& ORNSTEIN, P. H. The effect of induced eye movements on dreaming. Paper presented at the cighth meeting of the Association for the Psychophysiological Study of Sleep, Denver, March 1968.

DECKERT, G. H. Pursuit eye movements in the absence of a moving stimulus. Science, 1964 , 143, 1192-1193.

GAITO, J. Statistical dangers involved in counter balancing. Psychological Reports, 1958, 4. 463-468.

GAlTO, J. Repeated measurements design and counter balancing. Psychological Bulletin, $1961,58,45-54$.

GAARDER, K. Some patterns of fixation saccadic eye movements. Psychonomic Science, 1967, 7, 145-146.

HEBB, D. O. Organization of behavior. New York: Wiley, 1949.

HEBB, D. O. Concerning imagery. Psychological Review, 1968, 75, 466-477.

LORENS, S. A., \& DARROW, C. W. Eye movements, EEG, ESR, and EKG during mental multiplications. Electroencephalography \& Clinical Neurophysiology, 1962, 14, 739-746.

NORTON, D. W. An empirical investigation of some effects of non-normality and heterogeneity on the F-distribution. $\mathrm{PhD}$ thesis in Education, State University of Iowa, 1952. Cited in E. F. Lindquist. Design and analysis of experiments in psychology and education. Boston: Houghton Mifflin, 1953.

PAIVIO, A. Latency of verbal associations and imagery to noun stimuli as a function of abstractness and generality. Canadian Journal of Psychology, 1966, 20, 378-387.

PAIVIO, A., YUILLE, J., \& MADIGAN, S. Concreteness, imagery, and meaningfulness values for 925 nouns. Journal of Experimental Psychology Monograph Supplement, 1968, 76, No. 1, Part 2.

SIMPSON, H. M., \& PAIVIO, A. Effects on pupil size of manual and verbal indicators of cognitive task fulfillment. Perception \& Psychophysics, 1968, 3, 185-190.

YUILLE, J. C. Effects of noun imagery and meaningfulness on latencies of mediators and learning. Unpublished MA thesis, University of Western Ontario, London, Canada, 1965.

YUILLE, J. C., \& PAIVIO, A. Imagery and verbal mediation instructions in paired-associate learning. Journal of Experimental Psychology, $1968,78,436-441$.

ZIGMUND, V. Oculomotor activity during visual imagery of a moving stimulus pattern. Studia Psychologica, 1966, 4, 254-274.

\section{NOTES}

1. Baldridge, Whitman, Kramer, and Ornstein (1968) have recently used a similar procedure in the study of REMs and dreaming.

2. During Experiment 1 some Ss failed to push the button during the imaging period and, subsequently, gave a vividness rating of 5 , indicating they had not been able to generate an image to that particular noun pair. A maximum value of $20 \mathrm{sec}$ (the duration of the imaging period) was assigned as the imaginal latency in those cases in which a buttonpress did not occur. The result of this was that the mean scores for some Ss were spuriously high, due to an extreme score for one or two imaging periods, yielding slightly skewed score distributions. Although under some circumstances violations of the assumptions of the $F$ test do not affect the probability values (Norton, 1952), it was, nevertheless, considered wise to reanalyze the data: To determine if the distribution affected the results of the previous analyses, they were repeated using the median scores for each $S$ rather than the means. Briefly, the results of the analyses based on median scores were highly similar to those based on the mean scores. Indeed, a further analysis of the latency data based on reciprocal transformation of the scores also yielded similar effects, indicating that the results were not an artifact of the characteristics of the data. 
3. A distribution plot of the raw data revealed that the scores were not normally distributed, i.e., they were slightly negatively skewed. However, the Hartley test for heterogeneity of variance applied to the variance between groups yielded an $F \max =1.78$, indicating that the variances could be considered homogeneous.

4. Again a distribution plot of the raw data showed the scores were not normally distributed.

However, the $F \max =2.08$ was not significant; thus, the assumption of homogeneity of variance was met.

(Accepted for publication July 6, 1970.) 